\title{
Erradicación de Helicobacter pylori con la terapia triple de primera línea, 10 días versus 14 días
}

\section{Eradication of Helicobacter pylori with first-line triple therapy, 10 days versus 14 days}

\author{
María Nelly Manrique-Lemus', \\ José Luis Rojas-Vilca², \\ Mario Salomón Valdivia-Roldán ${ }^{3}$ y \\ Alaciel Melissa Palacios-Guillén ${ }^{4}$
}

Manrique-Lemus M, Rojas-Vilca J,Valdivia-Roldán M, Palacios-Guillén A. Erradicación de Helicobacter pylori con la terapia triple de primera línea, 10 días versus 14 días. Rev Soc Peru Med Interna. 2020;33(4): |38-| 45. https://doi.org/10.36393/spmi.v33i4.560

\begin{abstract}
RESUMEN
Objetivo: Determinar si la tasa de erradicación de Helicobacter pylori con la terapia triple de primera línea durante 14 días es superior que con 10 días. Material y métodos: Estudio de cohortes retrospectivo. Se revisaron las historias clínicas de pacientes con dispepsia y endoscopia digestiva alta con biopsia positiva a $\mathrm{H}$. pylori, quienes recibieron terapia triple con amoxicilina, claritromicina y omeprazol durante 10 ó 14 días. La erradicación se definió con la prueba del aliento I3C-UBT, 4 a 6 semanas post tratamiento. Resultados: Se estudió 38I pacientes, 207 recibieron tratamiento 10 días y 174 recibieron 14 días. La edad promedio fue $49 \pm 13,9$ (I5 a 82) años. Predominó el sexo femenino (69,8\%). La tasa de erradicación de $\mathrm{H}$. pylori en el grupo de tratamiento de 10 días y 14 días fue $79,7 \%$ y $82,2 \%$ respectivamente (riesgo relativo, I,03; intervalo de confianza 95\%, 0,94-I, I4; $\mathrm{p}=0,54 \mathrm{I}$ ). Los eventos adversos fueron reportados en el $75.1 \%$ y $77.3 \%$ de los regímenes de 10 y 14 días respectivamente $(p=0,634)$. Conclusión: La tasa de erradicación de $\mathrm{H}$. pylori con terapia triple de primera línea durante 14 días no fue superior a 10 días y las tasas de erradicación fueron mala e inaceptable respectivamente.
\end{abstract}

Palabras claves: Helicobacter pylori, tratamiento, dispepsia.

\section{ABSTRACT}

Objective: To determine if the eradication rate of Helicobacter pylori with first line triple therapy for 14 days is better than with 10 days. Material and methods: Retrospective cohort study. It was reviewed medical records of the patients with dyspepsia and upper digestive endoscopy with a positive $H$. pylori biopsy, who received triple therapy with amoxicillin, clarithromycin and omeprazole for 10 or 14 days. Eradication was defined by the I3C-UBT breath test, 4 to 6 weeks after treatment. Results:

I Médico gastroenteróloga e internista. Hospital EsSalud Uldarico Rocca Fernández, Villa El Salvador, Lima, Perú

2 Médico cirujano. Magister en Epidemiología Clínica. Facultad de Medicina Alberto Hurtado, Universidad Peruana Cayetano Heredia, Lima, Perú.

3 Médico gastroenterólogo. Clínica Centenario Peruano Japonesa, Lima, Perú.

4 Médico nefróloga. Hospital Nacional Daniel Alcides Carrión del Callao, Lima, Perú
It was evaluated 381 patients, 207 received treatment for 10 days and 174 for 14 days. The average age was $49 \pm 13.9$ (15 to 82$)$ years. The female sex was predominating $(69,8 \%)$. The eradication rate of $\mathrm{H}$. pylori in the 10-day and I4-day treatment group was $79,7 \%$ and $82,2 \%$ respectively (risk ratio, $1.03 ; 95 \%$ confidence interval, 0.94-I.I4; $p=0,54 I$ ). Adverse events were reported in $75,1 \%$ and $77,3 \%$ of the 10 and 14 days group respectively $(p=0,634)$. Conclusion: First-line triple therapy for 14 days for $H$. pylori eradication was not better than 10 days' therapy. The eradication rate was poor and unacceptable respectively.

Keywords: Helicobacter pylori, treatment, dyspepsia.

\section{INTRODUCCIÓN}

Helicobacter pylori (H. pylori) establece una infección crónica en la mucosa gástrica humana, resultando en una variedad de enfermedades gastroduodenales que van desde 
gastritis superficial y úlcera péptica a cáncer gástrico y linfoma MALT (tejido linfoide asociado a la mucosa). ${ }^{1}$

La infección por $H$. pylori es el factor de riesgo más frecuente para el desarrollo del cáncer gástrico, considerado carcinógeno tipo I. Por tanto, su eliminación es la estrategia más prometedora para reducir la incidencia de cáncer gástrico que representa la tercera causa de muerte por cáncer en el mundo. ${ }^{2,3}$ En el Perú el cáncer gástrico es la segunda neoplasia más frecuente en varones y la tercera en mujeres, representando la primera causa de muerte por cáncer en ambos sexos. ${ }^{4}$ Además, la erradicación de $H$. pylori evita la progresión de la gastritis crónica a lesiones premalignas (gastritis atrófica, metaplasia intestinal) al constituirse como medida principal de tratamiento en pacientes con enfermedad ulcero-péptica asociada a H. pylori. ${ }^{5-7}$

La dispepsia es un problema común en el mundo, en Estados Unidos la prevalencia aproximada es de $25 \%{ }^{8}$ En una comunidad de la selva de Perú se ha descrito la prevalencia hasta de $37,6 \%{ }^{9}$

El estándar para el diagnóstico de dispepsia funcional según los criterios de Roma III consisten en sensación de dolor o quemazón en el epigastrio, saciedad temprana, plenitud postprandial, o una combinación de estos síntomas. ${ }^{10} \mathrm{La}$ prevalencia global de dispepsia funcional en la comunidad de acuerdo a esta definición es entre 5-11\%., aunque el 5\% de los casos de dispepsia en la comunidad son atribuibles a la infección por H. Pylori. ${ }^{11,12}$ El efecto de la terapia erradicadora sobre los síntomas de dispepsia funcional es moderado, y los modelos económicos sugieren que la terapia erradicadora es una estrategia costo-efectiva para el manejo de la dispepsia funcional. . $^{13,14}$

Se ha observado que la eficacia de la terapia triple de primera línea (TTP) para la erradicación de $H$. pylori está disminuyendo debido, principalmente, al aumento de la resistencia a los antibióticos por lo que una de las estrategias es prolongar la terapia a 14 días. ${ }^{15}$

La resistencia a la claritromicina es el principal factor de riesgo de falla al tratamiento. En una revisión sistemática se reportó una disminución del $53 \%$ en la eficacia de erradicación cuando se utiliza esquemas con claritromicina en presencia de resistencia a este antibiótico. Por lo tanto, en lugares donde la resistencia a claritromicina es $<20 \%$ todavía se recomienda la terapia triple con amoxicilina, claritromicina y omeprazol. ${ }^{7,16}$

Con la finalidad de simplificar el tratamiento con menos efectos adversos y mayor cumplimiento de los pacientes se ha propuesto acortar el tiempo de tratamiento. La mayoría de estudios soportan que la terapia triple por 1 o 2 semanas están asociadas con tasas de erradicación similares. ${ }^{17-20}$ Sin embargo, la superioridad de la terapia triple por dos semanas sobre la terapia triple por una semana ${ }^{21}$ y sobre la terapia secuencial ${ }^{22}$ ha sido confirmada por otros estudios ${ }^{23}$ y recomendada por últimos consensos. ${ }^{24,25}$

La duración del tratamiento es un tema controversial pero no hay grandes diferencias entre los tratamientos de 7,10 , y 14 días, mientras que la diferencia de costos puede ser sustancial. ${ }^{16}$
El objetivo del estudio fue determinar si la tasa de erradicación de $H$. pylori con la terapia triple de primera línea durante 14 días era superior que con 10 días.

\section{MATERIAL Y MÉTODOS}

Diseño del estudio: Estudio observacional, analítico de tipo cohortes retrospectivo

Población: Se revisaron y evaluaron las historias clínicas de pacientes dispépticos ambulatorios atendidos en el servicio de gastroenterología del Hospital EsSalud Uldarico Rocca Fernández (HURF) de Villa El Salvador, del 2 de enero del 2012 al 30 de diciembre de 2014.

Criterios de inclusión: Pacientes con dispepsia, mayores de 15 años, con infección por $H$. pylori confirmada por biopsia endoscópica y con indicación de terapia triple antibiótica, con prueba de urea $-{ }^{13} \mathrm{C}$ espirada para detección de $H$. pylori 30 días post tratamiento.

\section{Grupo expuesto}

Indicación de TTP con amoxicilina $1000 \mathrm{mg}$ BID + claritromicina $500 \mathrm{mg}$ BID + omeprazol $20 \mathrm{mg}$ BID durante 14 días.

\section{Grupo no expuesto}

Indicación de TTP con amoxicilina $1000 \mathrm{mg}$ BID + claritromicina $500 \mathrm{mg}$ BID + omeprazol $20 \mathrm{mg}$ BID durante 10 días.

Criterios de exclusión: (a) tratamiento previo anti H. pylori, (b) tratamiento incompleto, (c) gastrectomía previa y (d) diagnóstico de cáncer gástrico

Muestra: El tamaño muestral se calculó en 172 pacientes para cada grupo de tratamiento, con tamaño total de la muestra de 344. Se usó el programa en línea OPEN EPI versión 3, considerando una razón de riesgo de 1.1 y potencia de $80 \%$, para una razón expuestos / no expuestos de 1.

Procedimientos y técnicas: En la revisión de las historias clínicas, se identificó pacientes con diagnóstico de infección por $H$. pylori que hayan recibido TTP con amoxicilina + claritromicina + omeprazol por 10 o 14 días.

La Infección por $H$. pylori $(1+, 2+, 3+)$ se determinó por biopsia gástrica (dos de antro y dos de cuerpo, en curvatura mayor y menor), que fueron analizados por anatomopatólogos del Hospital Nacional EsSalud Edgardo Rebagliati Martins en pacientes a quienes se les realizó endoscopia digestiva alta (con indicación de suspender ranitidina, omeprazol, bismuto y antibióticos 15 días antes del examen). Las muestras de biopsias fueron estudiadas con hematoxilina-eosina y se utilizó la Escala Visual Análoga del sistema actualizado de Sydney para graduar las variables histológicas de la gastritis. ${ }^{26}$

Se usó como estándar de diagnóstico de infección por H. pylori la presencia de la bacteria en cualquiera de las biopsias gástricas. Para el estadio de la gastritis con más de un diagnóstico histopatológico se eligió el más severo. Se incluyó en el estudio pacientes a quienes se les realizó 4-6 semanas post tratamiento la prueba de urea- ${ }^{13} \mathrm{C}$ espirada ${ }^{13}$ (C-UBT) usando una solución bebible de urea marcada con $\mathrm{C}^{13}$, se aseguró que los pacientes no habían recibido 
medicación como inhibidor de bomba de protones (IBP), subsalicilato de bismuto, ranitidina ni antibióticos 4 semanas antes del examen.

Se definió erradicación de H. pylori si la ${ }^{13} \mathrm{C}$-UBT fue negativa.

La recolección de datos se realizó en una ficha que incluyó datos como: sexo, edad, lugar de procedencia, diagnóstico endoscópico, estudio histopatológico, síntomas predominantes pre tratamiento, esquemas de tratamiento recibido, resultado de ${ }^{13} \mathrm{C}$-UBT para la detección de $H$. pylori y eventos adversos reportados durante el tratamiento. Análisis estadístico: Todos los datos de las fichas de recolección fueron introducidos en el programa Microsoft Excel 2016. El análisis estadístico se realizó con el paquete estadístico STATA v. 14.0. En el análisis descriptivo las variables categóricas son presentadas en tablas de frecuencias absolutas y relativas. Se evaluó la normalidad de la distribución de la variable numérica edad mediante la prueba de Shapiro-Wilk y se describió mediante su tendencia central y dispersión (promedio y desviación estándar por tener distribución normal). Se realizó análisis inferencial bivariado para evaluar la diferencia entre los grupos de tratamiento utilizando la prueba Chi-cuadrado para las variables categóricas y la prueba t-Student para la variable continua (edad) con distribución normal. Para evaluar la posible confusión de las variables intervinientes que alcanzaron diferencia estadísticamente significativa en el análisis bivariado se utilizó el Método de MantelHaenszel.
Para establecer la diferencia del efecto (erradicación de $H$. pylori) entre el grupo de expuestos (14 días) y no expuesto (10 días) se calculó la incidencia en cada grupo y el riesgo relativo (RR). Para el análisis inferencial multivariado se utilizó modelos lineales generalizados (GLM).

Consideraciones éticas: El estudio contó con la aprobación del Comité de Ética de la Red Desconcentrada RebagliatiEsSalud y del Comité Institucional de Ética en Humanos de la Universidad Peruana Cayetano Heredia, de manera previa a su inicio, preservando los principios éticos, según las normas internacionales para pacientes adultos.

La información de los pacientes fue recolectada de las historias clínicas e ingresadas en la base de datos de manera codificada, siendo su uso anónimo. No se solicitó consentimiento informado para la publicación del presente estudio, debido a que en este artículo no se publica ningún dato personal para identificar al paciente.

\section{RESULTADOS}

\section{Pacientes}

Entre enero 2012 y diciembre de 2014, 418 pacientes con algún síntoma dispéptico infectados con $H$. pylori recibieron TTP, 225 recibieron terapia triple durante 10 días y 193 durante 14 días. En el grupo de 10 días se encontró que 18 pacientes no completaron el estudio (13 completaron tratamiento, pero no tenían resultado de ${ }^{13} \mathrm{C}-\mathrm{UBT}$, en 4 se indicó tratamiento y no regresaron, 1 recibió tratamiento por 5 días) y en el grupo de 14 días se encontró que 19 pacientes no completaron el estudio (17 pacientes habían

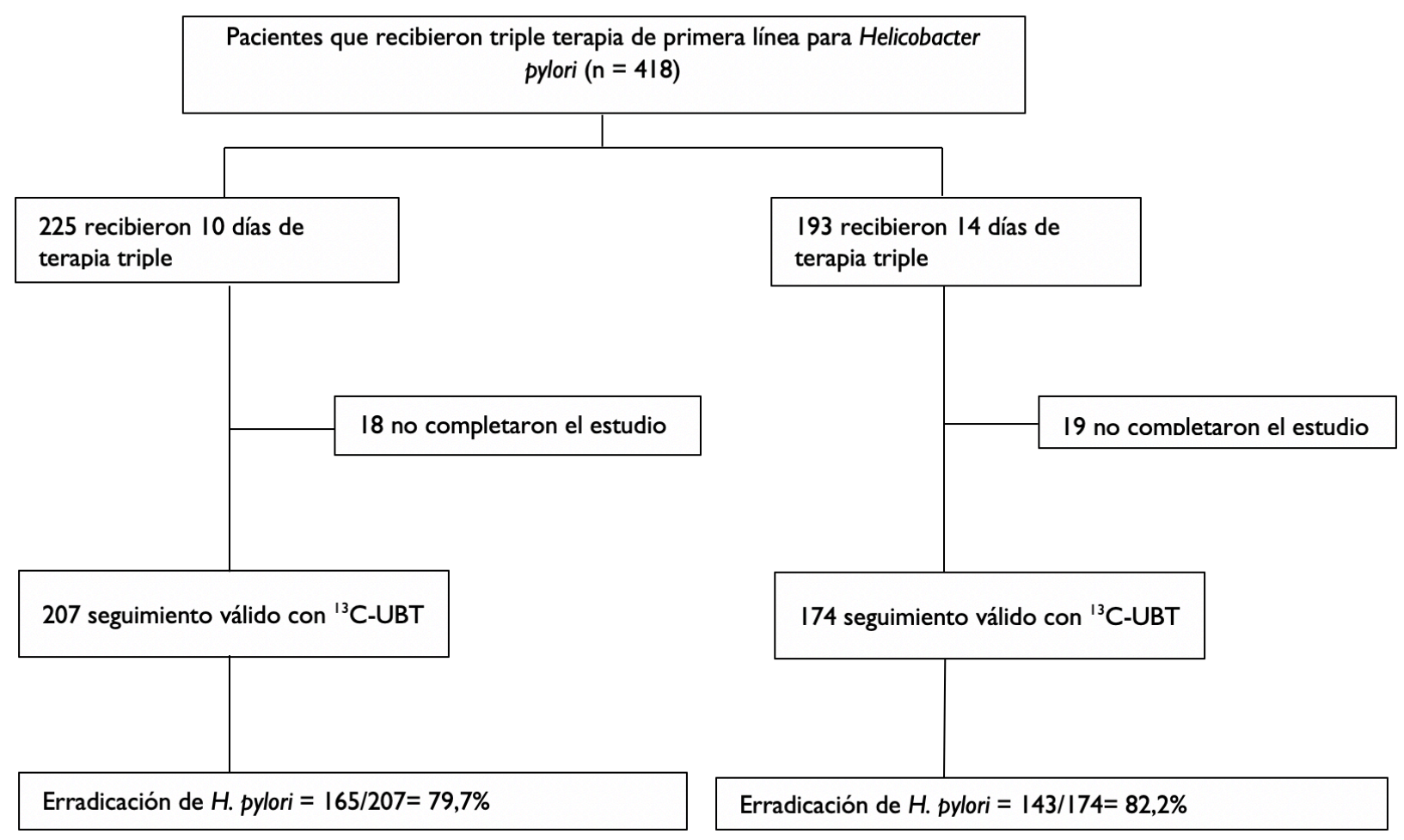

${ }^{13} \mathrm{C}-U B T$, Prueba de urea espirada usando una solución bebible urea marcada con $\mathrm{CI} 3$

Figura I. Diagrama de flujo de los pacientes incluidos en el estudio. Hospital EsSalud Uldarico Rocca Fernández, Lima- Perú, 20 I2-20 I4. 
completado el tratamiento pero no tenían resultado de ${ }^{13} \mathrm{C}$-UBT y 2 habían recibido tratamiento por 6 días). Finalmente, los grupos estuvieron constituidos por $207 \mathrm{y}$ 174 pacientes para los grupos que recibieron tratamiento por 10 y 14 días respectivamente (Figura 1).

La edad media y desviación estándar fue $49 \pm 13,9$, rango de 15 a 82 años. Se apreció un predominio del sexo femenino $(69,8 \%)$ sobre el masculino $(30,2 \%)$.

En el análisis bivariado de las características de base, los dos grupos de tratamiento fueron comparables en términos de sexo, edad, lugar de procedencia, diagnóstico endoscópico y principales síntomas dispépticos ( $\mathrm{p}>0,05)$. Se encontró diferencia significativa en la densidad de $H$. pylori $(\mathrm{p}<0,05)$ y en el estadio de la gastritis $(\mathrm{p}<0,01)$ (Tabla 1).

Erradicación de Helicobacter pylori: La tasa de erradicación de H. pylori en el grupo de tratamiento de 14 días fue $82,2 \%$ y en el grupo de tratamiento de 10 días fue $79,7 \%$. No se observó diferencia estadísticamente significativa en la comparación de las tasas de erradicación entre los dos grupos de tratamiento $(\mathrm{p}=0,541)$.

El RR para la erradicación de H. pylori con el régimen de

Tabla I. Características clínicas y demográficas de los pacientes con infección por Helicobacter pylori según régimen de tratamiento. Hospital EsSalud Uldarico Rocca Fernández, Lima- Perú, 20I2-20I4 ( $\mathrm{n}=38 \mathrm{I})$

\begin{tabular}{|c|c|c|c|}
\hline Variable & $\begin{array}{l}\text { Régimen de } 10 \text { días } \\
(\mathrm{n}=207) \\
\mathrm{n}(\%)\end{array}$ & $\begin{array}{l}\text { Régimen de } 14 \text { días } \\
(\mathrm{n}=174) \\
\mathrm{n}(\%)\end{array}$ & Valor $\mathrm{p}$ \\
\hline $\begin{array}{l}\text { Sexo } \\
\text { Masculino } \\
\text { Femenino }\end{array}$ & $\begin{array}{l}62(20,0) \\
145(70,1)\end{array}$ & $\begin{array}{l}53(30,5) \\
121(69,5)\end{array}$ & 0,914 \\
\hline $\begin{array}{l}\text { Edad (años) } \\
\text { Media } \pm \text { DS } \\
15-29 \\
30-39 \\
40-49 \\
\geq 50\end{array}$ & $\begin{array}{l}48,9 \pm 14,5 \\
19(9,2) \\
38(18,4) \\
53(25,6) \\
97(46,9)\end{array}$ & $\begin{array}{l}49,3 \pm 13,3 \\
8(4,6) \\
35(20,1) \\
45(25,9) \\
86(49,4)\end{array}$ & 0,772 \\
\hline $\begin{array}{l}\text { Lugar de procedencia } \\
\text { Villa El Salvador } \\
\text { San Juan de Miraflores } \\
\text { Villa María del Triunfo } \\
\text { Lurín } \\
\text { Otros }\end{array}$ & $\begin{array}{l}133(64,3) \\
35(16,9) \\
24(11,6) \\
11(5,3) \\
4(1,9)\end{array}$ & $\begin{array}{l}116(66,7) \\
19(10,9) \\
28(16,1) \\
7(4,0) \\
4(2,3)\end{array}$ & 0,366 \\
\hline $\begin{array}{l}\text { Diagnóstico endoscópico } \\
\text { Gastritis } \\
\text { Ulcera gástrica } \\
\text { Úlcera duodenal }\end{array}$ & $\begin{array}{l}20 \mathrm{I}(97, \mathrm{I}) \\
\mathrm{I}(0,5) \\
5(2,4)\end{array}$ & $\begin{array}{l}\text { I } 73(99,4) \\
0(0,0) \\
\text { I }(0,6)\end{array}$ & 0,226 \\
\hline $\begin{array}{l}\text { Densidad de } H \text {. pylori } \\
\text { Leve } \\
\text { Moderada } \\
\text { Severa }\end{array}$ & $\begin{array}{l}83(40,1) \\
78(37,7) \\
46(22,2)\end{array}$ & $\begin{array}{l}54(31,0) \\
60(34,5) \\
60(34,5)\end{array}$ & $\begin{array}{l}<0,05 \\
0,066 \\
0,518 \\
<0,01\end{array}$ \\
\hline $\begin{array}{l}\text { Estadio de la Gastritis } \\
\text { Gastritis crónica superficial } \\
\text { Gastritis crónica profunda } \\
\text { Metaplasia intestinal completa } \\
\text { Metaplasia intestinal incompleta } \\
\text { Displasia }\end{array}$ & $\begin{array}{l}\text { II } 3(54,6) \\
56(27,1) \\
12(5,8) \\
25(12,1) \\
1(0,5)\end{array}$ & $\begin{array}{l}93(53,5) \\
25(14,4) \\
22(12,6) \\
32(18,4) \\
2(1,2)\end{array}$ & $\begin{array}{l}<0,0 \text { I } \\
0,824 \\
<0,01 \\
<0,05 \\
0,085 \\
0,594\end{array}$ \\
\hline \multicolumn{4}{|l|}{ Principales síntomas dispépticos } \\
\hline Dolor epigástrico & $159(76,8)$ & I $43(82,2)$ & 0,198 \\
\hline Pesadez postprandial & $90(43,5)$ & $84(48,3)$ & 0,349 \\
\hline ERGE & $78(37,7)$ & $77(44,3)$ & 0,193 \\
\hline Flatulencia & $36(17,4)$ & $26(14,9)$ & 0,519 \\
\hline Halitosis & $25(12, I)$ & $13(7,5)$ & 0,135 \\
\hline Dolor en CSD & II $(5,3)$ & $16(9,2)$ & $0,14 \mid$ \\
\hline Diarrea & $12(5,8)$ & II $(6,3)$ & 0,830 \\
\hline Borborigmos & $8(3,9)$ & $2(1,2)$ & 0,118 \\
\hline Saciedad temprana & $3(1,5)$ & $2(1,2)$ & $\mathrm{I}, 008$ \\
\hline
\end{tabular}

CSD, cuadrante superior derecho de abdomen

*Se usó la prueba Chi-cuadrado y prueba exacta de Fisher para comparar las variables categóricas y la prueba t-Student para comparar la variable continua (edad). 
14 días comparado con 10 días fue 1,03 (IC 95\%: 0,941,14) (Tabla 2).

Al analizar la relación entre los regímenes de tratamiento de 10 y 14 días y la tasa de erradicación de $H$. pylori con las variables que alcanzaron diferencia estadísticamente significativa en el análisis bivariado (la densidad de H. pylori y el estadio de la gastritis) utilizando el Método de MantelHaenszel, no se encontró diferencia estadísticamente significativa entre los estimados crudos y ajustados de las dos variables $(p>0,05)$.
En el análisis multivariado utilizando el método de modelos lineales generalizados (GLM) para evaluar el efecto de factores pronósticos potenciales en la erradicación de H. pylori, como la densidad de $H$. pylori y el estadio de la gastritis que obtuvieron diferencia significativa en el análisis bivariado, el RR combinado no se modificó significativamente, manteniéndose como no diferencia en la erradicación entre los dos grupos $(\mathrm{p}=0,661)$ (Tabla 3).

La tasa global de erradicación con la TTP anti $H$. pylori en los pacientes que recibieron tratamiento por 10 días y 14 días fue de $80,8 \%$.

Tabla 2. Erradicación de Helicobacter pylori con la Terapia triple de primera línea con amoxicilina/claritromicina/ omeprazol. Hospital EsSalud Uldarico Rocca Fernández, Lima- Perú, 20I2-20I4 (n=38I)

\begin{tabular}{|c|c|c|c|c|c|c|}
\hline & \multicolumn{2}{|c|}{ Estatus de $H$. pylori después del tratamiento } & \multirow{2}{*}{ Total } & \multirow{2}{*}{$\begin{array}{l}\text { Tasa de } \\
\text { erradicación (\%) }\end{array}$} & \multirow{2}{*}{$\begin{array}{l}\text { RR, IC (95\%) } \\
\text { (Crudo) }\end{array}$} & \multirow{2}{*}{$P$} \\
\hline & Erradicado & No erradicado & & & & \\
\hline Régimen 14 días & 143 & 31 & 174 & 82,2 & \multirow{2}{*}{ I,03 $(0,94-1,14)$} & \multirow{2}{*}{0,541} \\
\hline Régimen 10 días & 165 & 42 & 207 & 79,7 & & \\
\hline
\end{tabular}

Tabla 3. Análisis multivariado para la erradicación de Helicobacter pylori.

\begin{tabular}{|c|c|c|}
\hline Variable & RR (IC 95\%) & $\mathrm{P}$ \\
\hline \multicolumn{3}{|l|}{ Tiempo de tratamiento } \\
\hline 10 días & Referencia & \\
\hline 14 días & $1,02(0,92-1,13)$ & 0,661 \\
\hline \multicolumn{3}{|l|}{ Densidad de $H$. pylori } \\
\hline Leve & Referencia & \\
\hline Moderada & $0,96(0,86-1,08)$ & $0,54 I$ \\
\hline Severa & $0,97(0,86-1,10)$ & 0,615 \\
\hline \multicolumn{3}{|l|}{ Estadio de la gastritis } \\
\hline Superficial y profunda & Referencia & \\
\hline Metaplasia y displasia & I,07 (0,95 - I,19) & 0,256 \\
\hline
\end{tabular}

Tabla 4. Eventos adversos en la triple terapia de Primera línea anti Helicobacter pylori según régimen de tratamiento. Hospital EsSalud Uldarico Rocca Fernández, Lima- Perú, 2012-2014 ( $=352$ )

\begin{tabular}{|c|c|c|c|c|c|}
\hline Variable & $\begin{array}{l}\text { Régimen de } 10 \text { días } \\
\mathrm{n}(\%)\end{array}$ & $\begin{array}{l}\text { Régimen de } 14 \text { días } \\
\mathrm{n}(\%)\end{array}$ & OR & IC (95\%) & Valor $\mathrm{p}$ \\
\hline Náuseas & $75(39,7)$ & $53(32,5)$ & 0,73 & $0,47-1,14$ & 0,163 \\
\hline Cefalea & $66(34,9)$ & $57(35,0)$ & 1,00 & $0,65-1,56$ & 0,992 \\
\hline Diarrea & $65(34,4)$ & $52(31,9)$ & 0,89 & $0,57-1,40$ & 0,621 \\
\hline Alteración del gusto & $42(22,2)$ & $42(25,8)$ & 1,22 & $0,74-1,99$ & 0,437 \\
\hline Distensión abdominal & $26(13,8)$ & $17(10,4)$ & 0,73 & $0,38-1,40$ & 0,342 \\
\hline Dolor abdominal & $19(10,1)$ & $10(6, I)$ & 0,59 & $0,26-1,30$ & 0,183 \\
\hline Mareos & $9(4,8)$ & $5(3, I)$ & 0,63 & $0,21-1,93$ & 0,586 \\
\hline Malestar general & II $(5,8)$ & $2(1,2)$ & 0,20 & $0,04-0,92$ & 0,025 \\
\hline Estreñimiento & $4(2, I)$ & $7(4,3)$ & 2,08 & $0,60-7,22$ & 0,358 \\
\hline Flatulencia & $3(1,6)$ & $5(3, I)$ & 1,96 & $0,46-8,34$ & 0,479 \\
\hline Borborigmos & $2(I, I)$ & $4(2,5)$ & 2,35 & $0,43-\mid 3,01$ & 0,421 \\
\hline ERGE & $2(1, I)$ & $2(1,2)$ & 1,16 & $0,16-8,34$ & 1,000 \\
\hline Vómitos & $0(0,0)$ & $2(1,2)$ & 0 & 0 & 0,214 \\
\hline
\end{tabular}




\section{Seguridad}

Los eventos adversos durante el tratamiento fueron reportados en el $75,1 \%$ de los pacientes que recibieron tratamiento por 10 días y en el $77,3 \%$ de los pacientes que recibieron tratamiento por 14 días, no hubo diferencia significativa entre los dos grupos $(\mathrm{p}=0,634)$.

Los eventos adversos más frecuentemente reportados fueron náuseas $(36,4 \%)$, cefalea $(34,9 \%)$, diarrea $(33,2 \%)$, alteración del gusto $(23,9 \%)$, distención abdominal $(12,2 \%)$ y dolor abdominal $(8,2 \%)$. No hubo diferencia significativa entre los dos regímenes de tratamiento $(\mathrm{p}>0,05)$ (Tabla 4$)$.

\section{DISCUSIÓN}

El presente estudio de cohorte retrospectivo mostró que la tasa global de erradicación de $H$. pylori con la TTP durante 10 y 14 días fue $80,8 \%$, tasa inferior a la reportada por otros estudios peruanos en años previos como el realizado por Rodríguez y col. en el 2003, que encuentra que los porcentajes de erradicación de la infección por H. pylori con la terapia triple con amoxicilina+ claritromicina + omeprazol administrado durante 7 días y 10 días no eran diferentes correspondiendo a $86.1 \%$ para ambos grupos (31/36), encontrándose que el $91.8 \%$ de las muestras fueron susceptibles a claritromicina (27).

En otro estudio realizado por Soto y colaboradores en el 2003 usando este mismo régimen durante 14 días la tasa de erradicación se elevó a 93\% (28). La disminución de la tasa de erradicación en nuestro estudio comparativamente a estos estudios anteriores podría deberse al aumento de la resistencia de la bacteria a la claritromicina.

En nuestra población la tasa de erradicación de $H$. pylori con el régimen de 14 y 10 días fue de $82,2 \%$ y de $79,7 \%$ respectivamente, sin mostrar diferencia estadísticamente significativa. Hallazgos similares se han reportado en Corea en el 2007, donde la terapia triple por 7 días (amoxicilina, claritromicina y omeprazol) no fue inferior a la terapia por 14 días (19).

En el estudio realizado por Park en el 2009, 107 pacientes con infección $H$. pylori fueron distribuidos en 4 grupos de tratamiento: grupos con IBP, amoxicilina y claritromicina durante 7,10 y 14 días. Otro grupo recibió la terapia secuencial por 10 días. Las tasas de erradicación fueron: $72,0 \%, 81,5 \%$ y $86,2 \%$ respectivamente. Y $80,8 \%$ en el grupo de terapia secuencial. No hubo diferencia significativa entre los cuatro grupos (20).

En el estudio de Choi realizado en Corea en el 2011, las tasas de erradicación de $H$. pylori con la terapia triple con rabeprazol, amoxicilina y claritromicina durante 7, 10 ó 14 días fueron según el análisis por intención de tratar (IT)/ análisis por protocolo (PP): 70,4\%/ 75,7\%, 74,7\%/81,9\% y $80,0 \% / 84,4 \%$ respectivamente. Y $75,6 \% / 82,0 \%$ para 10 días de terapia secuencial. Ni el análisis por IT o PP mostró diferencias significativas entre las tasas de erradicación con la terapia secuencial o con las terapias triple estándar ( $\mathrm{p}=0,416$ y $\mathrm{p}=0,405$ respectivamente) (29).

Estos estudios realizados en Corea fueron incapaces de proveer evidencia concluyente para la hipótesis que sostiene que prolongar el tratamiento por 2 semanas podría superar la resistencia a la claritromicina (30).

En el meta-análisis publicado por Yuan en el 2013 se encontró un incremento significativo en la tasa de erradicación de $H$. pylori entre la terapia triple por 14 días y 10 días (12 estudios, 84,4\% vs 78,5\%; RR: 0,72 (IC 95\%: 0,58-0,90); número necesario a tratar 17 (IC 95\%: 11 - 46). Sin embargo, el estudio fue de calidad moderada y la mayoría de información fue obtenida de estudios con alto riesgo de sesgos, especialmente la mayoría fueron no ciegos. Concluye que no está claro cuánto beneficio se gana o si hay más efectos adversos al incrementar la duración de 10 días a 14 días (23).

En el meta-análisis del 2014 publicado por Gong y col. en Corea la tasa global de erradicación con la terapia triple anti $H$. pylori fue de $74,6 \%$ (95\% IC, $72,1 \%-77,2 \%)$ con el análisis IT y $82 \%$ (IC 95\%, 80,8\%- 83,2\%) por PP. Las tasas de erradicación con tratamiento de 7 días y 14 días fueron $81,1 \%$ (IC 95\%, 79,8\%-82,3\%) y $85,3 \%$ (IC 95\%, $83,5 \%-87,1 \%$ ) por análisis PP, respectivamente. Concluye que la eficacia de la terapia triple para $H$. pylori es más baja que la deseada y que incrementar la duración de la terapia puede proveer efectividad en curar la infección, pero esta hipótesis necesita ser validada (30).

Es así que en el último Consenso de Florencia/ Maastricht $\mathrm{V}$ establece que la duración del tratamiento de la terapia triple basada en claritromicina e IBP debería ser extendida a 14 días, a menos que terapias más cortas hayan probado efectividad localmente (24).

El $H$. pylori es un agente infeccioso y el objetivo terapéutico de erradicación debería ser del $100 \%$ por lo que se ha establecido un umbral teórico de eficacia (excelente $>95 \%$, bueno $90-95 \%$, regular $85-89 \%$, malo $81-84 \%$ e inaceptable $\leq 80 \%$ ). Actualmente se considera aceptable acercarnos a superar el $90 \%$ de eficacia en el análisis por IT (31).

Por lo tanto, nuestros valores obtenidos con el régimen de 10 días $(79,7 \%)$ y 14 días $(82,2 \%)$ fueron inaceptables y malos respectivamente, resultados coherentes con la revisión sistemática publicada por Sierra y col. en el 2013 que evaluó la eficacia de las terapias actuales de primera y segunda línea, se encontró que las tasas de erradicación de la terapia triple estándar eran inaceptables $(\leq 80 \%)$ en países donde el $H$. pylori presenta alta resistencia a claritromicina y metronidazol y que un tiempo mayor a 7 días no mejoraba la eficacia de la terapia triple estándar (18).

Además, no se encontró asociación significativa entre el sexo, la edad, el lugar de procedencia, la densidad de $H$. pylori, el estadio de la gastritis, la enfermedad ulcerosa, los síntomas dispépticos y los efectos adverso en la erradicación de H. pylori. Lo cual guarda verosimilitud con estudios previos que muestran que la causa principal de falla a la TTP anti $H$. pylori es la resistencia a claritromicina (32) y (33).

Prolongar la TTP no mejoró la tasa de erradicación de $H$. pylori a niveles aceptables ( $>90 \%$ ) lo cual puede deberse a que existen otros factores importantes que deben ser 
evaluados en esta población, como son el estado genotípico y fenotípico del CYP2C19 (26) en estos pacientes; debiendo hacerse la variabilidad genética de $H$. pylori y la sensibilidad antimicrobiana (34), a fin de poder establecer un adecuado esquema de tratamiento para la erradicación de H. pylori. Estudios que no se realizaron por no estar disponibles en la práctica clínica.

Por otro lado se observó un predominio de efectos adversos en el grupo de los pacientes que recibieron tratamiento por 14 días $(77,3 \%)$ que en los que recibieron tratamiento por 10 días $(75,1 \%)$, sin embargo no fue significativo $(\mathrm{p}=$ $0,634)$.

Los eventos adversos reportados con mayor frecuencia fueron náuseas $(36,4 \%)$, cefalea $(34,9 \%)$, diarrea $(33,2 \%)$, alteración del gusto $(23,9 \%)$ y distención abdominal (12,2\%). Resultados comparables a los del estudio de Hyuk Soon Choi en el 2012, el evento adverso más frecuente fue náuseas y/o vómitos (58,3\%), seguido por distensión abdominal (50\%), discomfort epigástrico (41,7\%), diarrea $(41,7 \%)$, alteraciones del gusto $(16,7 \%)$ y prurito $(8,3 \%)$ (29).

En una última revisión realizada por Otero se reafirma que en cada país o región es necesario investigar esquemas de tratamiento y elegir los de mayor eficacia independientemente de los consensos, guías internacionales o meta-análisis (35).

Dentro de las limitaciones del presente estudio se menciona la naturaleza retrospectiva que incluye el sesgo de información y un tipo de sesgo de selección, el sesgo de referencia, ya que la población estuvo constituida por individuos que acuden a un hospital para atención por alguna dolencia.

Además, en el análisis comparativo bivariado se encontró diferencia significativa en la densidad de $H$. pylori y en el estadio de la gastritis, con los regímenes de tratamiento de 10 y 14 días, lo que podría sugerir un posible sesgo de indicación médica que podría influir sobre el resultado; sin embargo, al realizar el análisis multivariado no se encontró diferencia significativa o influencia de estas variables sobre la tasa de erradicación de $H$. pylori.

Por lo que, hasta que no se cuente con evidencia que determine la superioridad de la terapia triple durante 14 días sobre la de 10 días en la erradicación de H. pylori y seguridad, no se recomienda prolongar la terapia a 14 días; lo que confiere ventajas respecto a cumplimiento del tratamiento y costos.

En conclusión, la tasa de erradicación de H. pylori con terapia triple de primera línea durante 14 días no fue superior a 10 días y las tasas de erradicación fueron mala e inaceptable respectivamente. En el presente estudio, se confirma que incrementar la duración del tratamiento de 10 a 14 días no mejora la efectividad de la terapia triple de primera línea contra $H$. pylori a tasas aceptables ( $>90 \%)$; sin embargo, se encontró un aumento en la frecuencia de eventos adversos, aunque la diferencia no fue significativa. Es necesario el desarrollo de nuevos esquemas de tratamiento con mejor eficacia para erradicar el H. pylori en el Perú.

\section{REFERENCIAS BIBLIOGRÁFICAS}

1. Cao Q, Didelot X, Wu Z, et al. Progressive genomic convergence of two Helicobacter pylori strains during mixed infection of a patient with chronic gastritis. Gut. 2015; 64(4):554-61.

2. Malfertheiner P, Megraud F, O’Morain CA, et al. Management of Helicobacter pylori infection-the Maastricht IV/ Florence Consensus Report. Gut. 2012; 61(5):646-64.

3. Stewart BW, World Health Organization, International Agency for Research on Cancer, Organisation Mondiale de la Santé, World Health Organization, International Agency for Research on Cancer, et al. World cancer report. Lyon: IARC Press; 2014.

4. Perú, Ministerio de Salud. Análisis de la situación del cáncer en el Perú, 2013. Lima: Ministerio de Salud; 2013.

5. Leung WK, Lin S-R, Ching TYL, et al. Factors predicting progression of gastric intestinal metaplasia: results of a randomised trial on Helicobacter pylori eradication. Gut. 2004; 53(9):1244-9.

6. Correa P, Piazuelo BM, Wilson KT. Pathology of Gastric Intestinal Metaplasia: Clinical Implications: $A m$ J Gastroenterol. 2010; 105(3):493-8.

7. Ramírez Ramos A, Sánchez Sánchez R. Helicobacter pylori 25 años después (1983 -2008): epidemiología, microbiología, patogenia, diagnóstico y tratamiento. Rev Gastroenterol Perú. 2009; 29(2):158-70.

8. Talley NJ, Vakil N, the Practice Parameters Committee of the American College of Gastroenterology. Guidelines for the Management of Dyspepsia. Am J Gastroenterol. 2005;100(10):232437.

9. Curioso W, Donaires N, Bacilio C, et al. Prevalencia y asociación de la dispepsia y el síndrome de intestino irritable en una comunidad de la Selva Peruana. Rev Gastroenterol Perú 2002; 22(2):129-40.

10. Tack J, Talley NJ, Camilleri M, et al. Functional Gastroduodenal Disorders. Gastroenterology. 2006;130(5):1466-79.

11. Ford AC, Marwaha A, Sood R, et al. Global prevalence of, and risk factors for, uninvestigated dyspepsia: a meta-analysis. Gut 2015; 64(7):1049-57.

12. Moayyedi P, Forman D, Braunholtz D, et al. The proportion of upper gastrointestinal symptoms in the community associated with Helicobacter pylori, lifestyle factors, and nonsteroidal antiinflammatory drugs. Am J Gastroenterol. 2000; 95(6):1448-55.

13. Moayyedi P, Soo S, Deeks J, et al. Eradication of Helicobacter pylori for non-ulcer dyspepsia. Cochrane Database Syst Rev. 2006; 2:CD002096.

14. Moayyedi P, Soo S, Deeks JJ, et al. Systematic review and economic evaluation of Helicobacter pylori eradication treatment for nonulcer dyspepsia. BMJ. 2000; 321:659-64.

15. Egan B, Katicic M, O'Connor H, et al. Treatment of Helicobacter pylori. Helicobacter. 2007; 12(Suppl.1): 31-37

16. Hunt R., Megraud F, Bazzoli F, et al. Guías prácticas de la Organización Mundial de Gastroenterología: Helicobacter pylori en los países en desarrollo. Gastroenterol. latinoam 2010. 21(2):16581.

17. Fuccio L, Minardi M, Zagari R, et al. Meta-analysis: duration of first-line proton-pump inhibitor based triple therapy for Helicobacter pylori eradication. Ann Intern Med. 2007; 147(8):553-62.

18. Sierra F, Forero JD, Rey M. Tratamiento ideal del Helicobacter pylori: una revisión sistemática. Rev Gastroenterol México. 2014; 79(1):28-49.

19. Kim BG, Lee DH, Ye BD, et al. Comparison of 7-day and 14-day proton pump inhibitor-containing triple therapy for Helicobacter pylori eradication: neither treatment duration provides acceptable eradication rate in Korea. Helicobacter. 2007; 12(1):31-5.

20. Park S, Chun H, Kim E, et al. The 10-day sequential therapy for Helicobacter pylori eradication in Korea: less effective than expected [Abstract]. Gastroenterology. 2009; 136: A339-40.

21. Paoluzi P, Iacopini F, Crispino P, et al. 2-week triple therapy for Helicobacter pylori infection is better than 1-week in clinical practice: a large prospective single-center randomized study. Helicobacter. 2006; 11(6):562-8.

22. Greenberg ER, Anderson GL, Morgan DR, et al. 14-day triple, 5-day concomitant, and 10-day sequential therapies for Helicobacter 
pylori infection in seven Latin American sites: a randomised trial. Lancet Lond Engl. 2011; 378(9790):507-14.

23. Yuan Y, Ford AC, Khan KJ, et al. Optimum duration of regimens for Helicobacter pylori eradication. Cochrane Database Syst Rev. 2013; (12):CD008337.

24. Malfertheiner P, Megraud F, O'Morain CA, et al. Management of Helicobacter pylori infection - the Maastricht V/Florence Consensus Report. Gut. 2017; 66(1):6-30.

25. Fallone CA, Chiba N, van Zanten SV, et al. The Toronto Consensus for the Treatment of Helicobacter pylori Infection in Adults. Gastroenterology. 2016; 151(1):51-69.e14.

26. Dixon MF, Genta RM, Yardley JH, et al. Classification and grading of gastritis. The updated Sydney System. International Workshop on the Histopathology of Gastritis, Houston 1994. Am J Surg Pathol. 1996; 20(10):1161-81.

27. Rodríguez W, Pareja A, Yushimito L, et al. Omeprazol, amoxicilina y claritromicina en el tratamiento de Helicobacter pylori, en los regímenes de 7 y 10 días. RevGastroenterol Perú. 2003; 23(3): 17783.

28. Soto G, Bautista CT, Roth DE, et al. Helicobacter pylori Reinfection Is Common in Peruvian Adults after Antibiotic Eradication Therapy. J Infect Dis. 2003; 188(9):1263-75.

29. Choi HS, Chun HJ, Park SH, et al. Comparison of sequential and 7-, 10-, 14-d triple therapy for Helicobacter pylori infection. World $J$ Gastroenterol. 2012; 18(19):2377-82.

30. Gong EJ, Yun S-C, Jung H-Y, et al. Meta-Analysis of First-Line Triple Therapy for Helicobacter pylori Eradication in Korea: Is It Time to Change? J Korean Med Sci. 2014; 29(5):704.

31. Molina-Infante J, Corti R, Doweck J, et al. Avances recientes en el tratamiento de la infección por Helicobacter pylori. Acta Gastroenterol Latinoam. 2017; 47(1):75-85.
32. Molina-Infante J, Gisbert JP. Actualización de la eficacia de la terapia triple para la infección por Helicobacter pylori y de la resistencia a claritromicina en España (2007 - 2012). Gastroenterol Hepatol. 2013; 36 (6):375-381.

33. Muñoz N, Sánchez-Delgado J, Baylina M, et al. Prevalencia de las resistencias de Helicobacter pylori tras el fracaso de una primera línea de tratamiento. Revisión sistemática. Gastroenterol Hepatol. 2018; 41 (10):654-662.

34. Furuta T, Graham DY. Pharmacologic Aspects of Eradication Therapy for Helicobacter pylori Infection. Gastroenterol Clin North Am. 2010; 39(3):465-80.

35. Otero W, Gómez M, Otero L, et al. Helicobacter pylori: ¿cómo se trata en el 2018? Rev Gastroenterol Perú. 2018; 38(1):54-63.

Conflictos de interés: Los autores declaran no tener ningún conflicto de intereses

Financiamiento: No se recibió patrocinio de ningún tipo para realizar el presente artículo.

Agradecimientos: El autor agradece al grupo de gastroenterólogos y enfermeras endoscopistas del HURF por su cooperación en la realización del presente estudio y a la empresa DIVCOM S.A.C. por su apoyo en el HURF

\section{CORRESPONDENCIA:}

María Nelly Manrique Lemus, marianelly20@yahoo.es

Fecha de recepción: 02-II-2020.

Fecha de aceptación: 23-I|-2020 